\title{
The Failure Rate Experimental Study of Special CNC Machine Tool
}

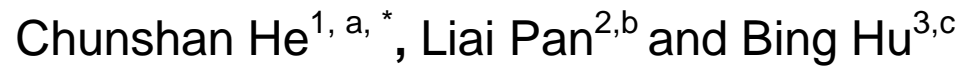 \\ ${ }^{1,2,3}$ College of Mechanical and Vehicle Engineering Changchun University,Changchun, China \\ aemail: he03cs@126.com, bemail: panli636@126.com, email: 1181725754@qq.com
}

Keywords: One-sample, Special CNC Machine tool, Failure Rate, Reliability of Special CNC Machine Tool.

Abstract. Based on one-sample special CNC machine tool, the failure distribution function and failure rate function, whose failure data have been analyzed by experiment, are determined in this paper. The special CNC machine tool time of which are in early failure or haphazard failure can be judged in terms of the failure rate function of it, and that the reliability of special CNC machine tool is improved by eliminating failures.

\section{Introduction}

Based on machine failure distribution function and reliability function, a special CNC machine tool failure rate of the single sample was given. How to establish the special NC machine tool failure distribution function is the key. In the paper, the time distribution model between failures has been determined taking the fitting experimental method, its correctness has been verified, and the reliability of the machine tool has been evaluated. Theory distribution function of fault interval time could be defined as:

$$
F(t)=P(T<t)
$$

In the formula, $T$ was interval time of failure in general; $t$ was interval time of any failure.

Assuming that $t_{1}, t_{2}, \ldots t_{k}$ were observed values of interval time of failure, by this group of observed values, order statistic of interval time of failure were obtained such as $t_{(1)}, t_{(2)}, \ldots t_{(k)}$. Then the empirical distribution function of interval time of $\mathrm{CNC}$ machine tool failure interval time was following as:

$$
F_{(k)}(t)= \begin{cases}0, & t<t_{(1)} \\ i / k, & t_{(i)} \leq t<t_{(i+1)}, i=1,2, \mathrm{~L} \mathrm{~L} k \\ 1, & t \geq t_{(k)}\end{cases}
$$

By the empirical distribution function $\mathrm{F}_{(\mathrm{k})}(\mathrm{t})$, theory distribution function $\mathrm{F}(\mathrm{t})$ could be estimated. And by $F(t)$ shape, the shape of a probability density function $f(t)$ of failure interval time could be judged. So by the $F_{(k)}(t)$ shape, the $f(t)$ shape could be preliminary judged. Graphics $F_{(k)}(t)$ was stepped line chart to fit the graphics in a row, to simplify the type (2) as follows:

$$
F_{(k)}(t)=i / k \quad i=1,2, \mathrm{~L} \mathrm{~L} k
$$

According to the type (3), $\mathrm{F}_{(\mathrm{k})}(\mathrm{t})$ has been fitted, so observed values of failure interval time $\mathrm{t} \in$ [and] have been divided into $\mathrm{n}$ groups. The abscissa was median of each group time, the ordinate was cumulative frequency of each group, thus make a $\mathrm{F}_{(\mathrm{k})}(\mathrm{t})$ scatter plot. According to the scatter plot, the empirical distribution function $\mathrm{F}_{(\mathrm{k})}(\mathrm{t})$ of failure interval time could be determined to convex or concave. And then judge that the distribution of the failure interval time was normal distribution or lognormal distribution, exponential distribution or Weibull distribution. Suppose the failure interval time obeyed a certain distribution, and then by linear regression analysis, the parameters of the distribution have been estimated. For fitting effect of the distribution, the linear correlation test has been adopted. If the linear correlation was established, it would introduce that the assuming distribution set up [1]. Finally distribution function failure interval time was $d$ tested, because the $\mathrm{d}$ test was better than the $\chi^{2}$ test.

The $\mathrm{d}$ test is that $\mathrm{k}$ test data are arranged according to the order from small to large; according to the hypothesis, calculate the distribution $\mathrm{F}_{0}\left(\mathrm{x}_{\mathrm{i}}\right)$ of each corresponding data, and compared with the empirical distribution function $\mathrm{F}_{\mathrm{k}}\left(\mathrm{x}_{\mathrm{i}}\right)$ to determine the largest absolute value of difference which is the 
observed value $D_{k}$ of test statistics. Compare $D_{k}$ with the critical value $D_{k, a}$. Satisfy the following conditions, then accept the null hypothesis, or reject the null hypothesis:

$$
D_{k}=\sup _{-\infty<x<+\infty}\left|F_{k}(x)-F_{0}(x)\right|=\max \left\{d_{i}\right\} \leq D_{k, \alpha}
$$

In the formula, $\mathrm{F}_{0}(\mathrm{x})$ was The original hypothesis distribution function; $\mathrm{F}_{\mathrm{k}}(\mathrm{x})$ was Empirical distribution function; $\mathrm{D}_{\mathrm{k}, \mathrm{a}}$ was the critical value.

$$
d_{i}=\max \left\{F_{0}\left(x_{i}\right)-\frac{i-1}{k}, \frac{i}{k}-F_{0}\left(x_{i}\right)\right\}
$$

After inspection, probability density function $\mathrm{f}(\mathrm{t})$ and distribution function $\mathrm{F}(\mathrm{t})$ of failure interval time could be determined. And then determine reliability function and failure rate function of the machine tool.

\section{Failure Rate Function of the Single Sample Special NC Machine Tool}

If $\mathrm{T}$ was continuous random variable, it said machine trouble-free working time, so the probability of reliable work at the time $t$ was following: $R(t)=P(T>t)$

The probability of machine tool failure was: $F(t)=P(T \leq t)$

$\mathrm{F}(\mathrm{t})$ was a random variable distribution function, the density function was: $f(t)=\frac{d}{d t} F(t)$

$\mathrm{F}(\mathrm{t})$ was unconditional probability density;

$\lambda(\mathrm{t})$ was known as the failure rate function, was the conditional probability density:

$$
\lambda(t)=\lim _{\Delta t \rightarrow 0} \frac{1}{\Delta t} P(t<T \leq t+\Delta t \mid T>t)=\lim _{\Delta t \rightarrow 0} \frac{1}{\Delta t} \frac{P[(t \mathrm{p} T \leq t+\Delta t) \mid T \mathrm{f} t]}{P(T \mathrm{f} t)}=\lim _{\Delta t \rightarrow 0} \frac{1}{\Delta t} \frac{f(t) \Delta t}{R(t)}=\frac{f(t)}{R(t)}
$$

The formula (9) was considered from the life of the unit [2], so it was in conformity with the actual requirements that the failure rate function of the single sample special $\mathrm{NC}$ machine tool was used $\lambda(\mathrm{t})$ to description.

\section{Determination of failure distribution function}

Now with a certain type of failure data of $\mathrm{CNC}$ machine tool as an example, the failure distribution function of the machine tool has been discussed. The observed values of failures interval time $t$ $[10.45,690.53]$ have been divided into 5 groups. The failure frequency and cumulative frequency were shown in table 1 .

Table.1. Failure frequency and cumulative frequency

\begin{tabular}{|c|c|c|c|c|c|c|}
\hline Group & $\begin{array}{c}\text { On the } \\
\text { interval }\end{array}$ & $\begin{array}{c}\text { Under the } \\
\text { range }\end{array}$ & $\begin{array}{c}\text { In the group } \\
\text { values }\end{array}$ & $\begin{array}{c}\text { frequency } \\
\text { number }\end{array}$ & frequency & Accumulative \\
\hline 1 & 10.45 & 146.47 & 78.46 & 5 & 0.3571 & 0.3571 \\
\hline 2 & 146.47 & 282.48 & 214.47 & 3 & 0.2143 & 0.5714 \\
\hline 3 & 282.48 & 418.50 & 350.49 & 3 & 0.2143 & 0.7857 \\
\hline 4 & 418.50 & 554.51 & 486.51 & 2 & 0.1429 & 0.9286 \\
\hline 5 & 554.51 & 690.53 & 622.52 & 1 & 0.0714 & 1.000 \\
\hline
\end{tabular}

To the abscissa denotes the value in each time the group, each group of probability density of the observed value as the ordinate.

The calculation $\hat{f}(t)$ was as follows:

$$
\hat{f}(t)=\frac{k_{i}}{k \Delta t_{i}}
$$

In the formula, $\mathrm{k}_{\mathrm{i}}$ was the failure frequency number in each group failure interval time; $\mathrm{k}$ was total failure frequency, for 14 times; $\Delta \mathrm{t}_{\mathrm{i}}$ was class width for $136.02 \mathrm{~h}$.

With the abscissa denotes the value in each time the group, each group of cumulative frequency as the ordinate, thus make a $\mathrm{F}_{(\mathrm{k})}(\mathrm{t})=i / k$ scatter diagram. 
The results show that the failures interval time the type of $\mathrm{CNC}$ machine tool was not subject to normal distribution or lognormal distribution, was the exponential distribution or Weibull distribution. Suppose the distribution was Weibull distribution, probability density function and distribution function of the Weibull distribution were:

$$
f(t)=\left\{\begin{array}{l}
\frac{\beta}{\alpha}\left(\frac{t-\gamma}{\alpha}\right)^{\beta-1} \exp \left[-\left(\frac{t-\gamma}{\alpha}\right)^{\beta}\right], t \geq \gamma \quad(11) \quad F(t)=\left\{\begin{array}{l}
\int_{0}^{t} f(t) d t=1-\exp \left[-\left(\frac{t-\gamma}{\alpha}\right)^{\beta}\right], t \geq \gamma \\
0, \quad t<\gamma
\end{array} \quad t<\gamma\right.
\end{array}\right.
$$

In the formula (11), (12), $\beta$ was the shape parameter, $\beta>0 ; \alpha$ was the scale parameter, $\alpha>0$; $\gamma$ was the position parameters, $\gamma>0$. Change $\gamma$ affected translational position of the probability density curve. Before $t=\gamma$, the product was not down; After $\mathrm{t}=\gamma$, the product failure.

In practice, often assume that when $\mathrm{t}=0$, product failure. So, types (11), (12) then were simplified as:

$$
f(t)=\frac{\beta}{\alpha}\left(\frac{t}{\alpha}\right)^{\beta-1} \exp \left[-\left(\frac{t}{\alpha}\right)^{\beta}\right], t \geq 0 \quad \text { (13) } \quad F(t)=1-\exp \left[-\left(\frac{t}{\alpha}\right)^{\beta}\right], \quad t \geq 0
$$

The distribution of failures interval time has been studied by Weibull distribution with two parameters.

\section{Fitting Inspection of Failure Distribution Function}

\section{Parameters Estimation of the Weibull Distribution}

Suppose that one-dimensional linear regression equation was:

$$
y=A+B x
$$

According to the two parameter Weibull distribution, to linear transformation type (14), available:

$$
y=\ln \ln \frac{1}{1-F(t)} \quad(16), \quad x=\ln t \quad(17), \quad A=-\beta \ln \alpha \quad(18), B=\beta
$$

If observed values of failure distribution function were $t_{1}, t_{2}, \ldots t_{k}$, according to the type (16), (17), the values were conversed into the $\mathrm{x}, \mathrm{y}$ in type (15). Intercept $\mathrm{A}$ and slope $\mathrm{B}$ of regression straight line could be obtained by the least squares. Thus two parameters $\alpha$, $\beta$ of Weibull distribution could be estimated by type (18), (19). Value $\mathrm{x}_{\mathrm{i}}$ could be calculate by type (17), namely

$$
x_{i}=\ln t_{i}
$$

Value $\mathrm{y}_{\mathrm{i}}$ could be calculated by type (16). Before calculation, the value $\mathrm{F}\left(\mathrm{t}_{\mathrm{i}}\right)$ should be estimated firstly. General use median estimated:

$$
\hat{F}\left(t_{i}\right) \approx \frac{i-0.3}{k+0.4}
$$

In this way, by the least squares method, it was available: $\hat{B}=\frac{l_{x y}}{l_{x x}} \quad$ (22), $\quad \hat{A}=\bar{y}-\hat{B} \bar{x}$

By type (18) and (19), available: $\hat{\beta}=\hat{B}$

$$
\hat{\alpha}=\exp (-\hat{A} / \hat{B})
$$

According to the failure data of $\mathrm{CNC}$ machine tool, two parameters Weibull distribution have been estimated. Failure data were shown in table 2. 
Table.2. Failure test data of a special NC machine tool

\begin{tabular}{|c|c|c|c|c|c|c|c|}
\hline $\mathrm{No}$ & $\mathrm{t}_{\mathrm{i}}$ & $\mathrm{x}_{\mathrm{i}}$ & $\mathrm{F}\left(\mathrm{t}_{\mathrm{i}}\right)$ & $\mathrm{y}_{\mathrm{i}}$ & $\mathrm{x}_{\mathrm{i}}^{2}$ & $\mathrm{y}_{\mathrm{i}}^{2}$ & $\mathrm{x}_{\mathrm{i}} \mathrm{y}_{\mathrm{i}}$ \\
\hline 1 & 11.45 & 2.44 & 0.0486 & -2.9991 & 5.99 & 8.9945 & -7.3118 \\
\hline 2 & 12.96 & 2.56 & 0.1181 & -2.0744 & 6.56 & 4.3033 & -5.3145 \\
\hline 4 & 15.37 & 2.73 & 0.1875 & -1.5720 & 7.47 & 2.4710 & -4.2952 \\
\hline 5 & 143.00 & 4.96 & 0.3264 & -0.9286 & 24.63 & 0.8623 & -4.6086 \\
\hline 6 & 160.03 & 5.08 & 0.3958 & -0.6859 & 25.76 & 0.4697 & -3.4785 \\
\hline 7 & 171.18 & 5.14 & 0.4653 & -0.4684 & 26.45 & 0.2194 & -2.4088 \\
\hline 8 & 236.27 & 5.46 & 0.5347 & -0.2627 & 29.87 & 0.0717 & -1.4631 \\
\hline 9 & 349.49 & 5.86 & 0.6042 & -0.0761 & 34.30 & 0.0058 & 0.4454 \\
\hline 10 & 389.27 & 5.96 & 0.6736 & 0.1130 & 35.57 & 0.0128 & 0.6741 \\
\hline 11 & 418.40 & 6.04 & 0.7431 & 0.3067 & 36.44 & 0.094 & 1.8512 \\
\hline 12 & 450.25 & 6.11 & 0.8125 & 0.5152 & 37.33 & 0.2654 & 3.1478 \\
\hline 13 & 516.95 & 6.25 & 0.8819 & 0.7592 & 39.04 & 0.5764 & 4.7435 \\
\hline 14 & 689.53 & 6.54 & 0.9514 & 1.1065 & 42.72 & 1.2244 & 7.2324 \\
\hline
\end{tabular}

By type (22) and (23), available:

$$
\hat{B}=0.77129, \hat{A}=-4.37678
$$

By (24) and (25), available: $\hat{\beta}=0.77129, \hat{\alpha}=291.4$

For fitting effect of Weibull distribution, the linear correlation test was used, so:

$$
\hat{\rho}=\frac{\sum_{i=1}^{k} x_{i} y_{i}-k \overline{x y}}{\sqrt{\left(\sum_{i=1}^{k} x_{i}^{2}-k \bar{x}^{2}\right)\left(\sum_{i=1}^{k} y_{i}{ }^{2}-k \bar{y}^{2}\right)}}
$$

When $|\rho|>\rho_{0}$, it was seen as $X$ and y linear correlation, which conformed to the Weibull distribution.

It was calculated in reliability of the information management system [3], $\hat{\rho}=0.9517$, When significant level $\alpha=0.1$, correlation coefficient value $\rho_{0}=1.645 /(k-1)^{1 / 2}=0.4562$.

For $|\hat{\rho}|>\rho_{0}$, we thought failure distribution function of the NC machine tool obeyed Weibull distribution. 


\section{Hypothesis Test of Weibull Distribution}

Hypothesis test data of a special NC machine tools were shown in table 3.

Table.3. Hypothesis test data of a special NC machine tools

\begin{tabular}{|c|c|c|c|c|c|}
\hline No & $\mathrm{t}_{\mathrm{i}}$ & $\mathrm{F}_{0}\left(\mathrm{t}_{\mathrm{i}}\right)$ & $(\mathrm{i}-1) / \mathrm{k}$ & $\mathrm{i} / \mathrm{k}$ & $\mathrm{d}_{\mathrm{i}}$ \\
\hline 1 & 11.45 & 0.079397 & 0 & 0.0714 & 0.00797 \\
\hline 2 & 12.96 & 0.086988 & 0.0714 & 0.1429 & 0.05587 \\
\hline 3 & 15.37 & 0.098575 & 0.1429 & 0.2143 & 0.11571 \\
\hline 4 & 100.66 & 0.356690 & 0.2143 & 0.2857 & 0.07098 \\
\hline 5 & 143.00 & 0.439016 & 0.2857 & 0.3571 & 0.08187 \\
\hline 6 & 160.03 & 0.46761 & 0.3571 & 0.4286 & 0.03904 \\
\hline 8 & 171.18 & 0.485718 & 0.4286 & 0.500 & 0.01482 \\
\hline 9 & 349.49 & 0.684358 & 0.5714 & 0.6429 & 0.04060 \\
\hline 10 & 389.27 & 0.71345 & 0.4629 & 0.7143 & 0.00083 \\
\hline 11 & 418.40 & 0.7332 & 0.7143 & 0.7857 & 0.05251 \\
\hline 12 & 450.25 & 0.752924 & 0.7857 & 0.8571 & 0.10422 \\
\hline 13 & 516.95 & 0.7888 & 0.8571 & 0.9286 & 0.1398 \\
\hline 14 & 689.53 & 0.85146 & 0.9286 & 1.000 & 0.14354 \\
\hline
\end{tabular}

Take a significance level $\alpha=0.10$, by empirical formula:

$$
D_{k, \alpha}=\frac{1.22}{\sqrt{n}}=0.3261
$$

For $\mathrm{D}_{\mathrm{k}}<\mathrm{D}_{\mathrm{k}, \mathrm{a}}$, receive the null hypothesis, that the average trouble-free working time of the machine obeyed Weibull distribution. The failure probability density function $\mathrm{f}(\mathrm{t})$ and distribution function $\mathrm{F}(\mathrm{t})$ were :

$$
f(t)=\frac{0.77}{291.4}\left(\frac{t}{291.4}\right)^{-0.23} \exp \left[-\left(\frac{t}{291.4}\right)^{0.77}\right] \quad(27), \quad F(t)=1-\exp \left[-\left(\frac{t}{291.4}\right)^{0.77}\right]
$$

\section{Failure Rate Function}

By type (9), failure rate function was:

$$
\lambda(t)=\frac{f(t)}{R(t)}=0.0026\left(\frac{t}{291.4}\right)^{-0.23}
$$

In the failure analysis of the product, $\beta$ was associated with the fault mechanism of the product, different $\beta$ values was with different fault mechanism. When the $\beta<1$, showing the life distribution of the early failure period; When the $\beta=1$, showing life distribution of the random failure period; When the $\beta>$ 1 , showing life distribution of the wear failure. $\alpha$ was associated with the load of working conditions, load was big, the corresponding $\alpha$ was small; And vice versa. From type (29) analysis show that $\beta<$ $1, \lambda(t)$ was downward trend, the special NC machine tools was in the early failure period when it was not 
appropriate for the customer to buy the machine. The machine should be continued to early failure experiments, formulated reasonable specification of early failure of the experiment. On the premise of no damage to the machine tool, as far as possible eliminate early failure; improve the reliability of machine tool.

If failure data were data samples of single failure, Bayesian estimation method should be adopted. Selecting incomplete $\beta$ distribution $B\left(\theta_{1}, \theta_{2}, a, b\right)$ as prior distribution was feasible; when $a \leq 1, b>1$, Bimperfect distribution density function was $\mathrm{p}_{\mathrm{i}}$ monotone decreasing function, accord that the possibility was large that failure probability $p_{i}$ was small, the possibility was small that failure probability $p_{i}$ was large, and then the parameters $a, b, \theta_{1}, \theta_{2}$, could be properly selected. For example $\mathrm{a}=1$, parameter $\mathrm{b}$ obeyed uniform distribution of interval $[1, \mathrm{C}], \theta_{2}=1, \theta_{1} \geq \mathrm{p}_{\mathrm{i}}$, the constant $\mathrm{C}$, according to the incomplete distribution $\beta$ density function $B\left(\theta_{1}, \theta_{2}, a, b\right)$.

$$
f\left(p_{i} ; \theta_{1}, \theta_{2}, a, b\right)=\frac{\left(p_{i}-\theta_{1}\right)^{a-1}\left(\theta_{2}-p_{i}\right)^{b-1}}{B(a, b)\left(\theta_{2}-\theta_{1}\right)^{a+b-1}} \quad\left(0<\theta_{1}<p_{i}<\theta_{2} \leq 1\right)
$$

The prior distribution formula has been determined for ${ }^{[4]}$ :

$$
h\left(p_{i} \mid p_{i-1}\right)=\frac{1}{C-1} \int_{1}^{C} \frac{\left(1-p_{i}\right)^{b-1}}{B(1, b)\left(1-p_{i-1}\right)^{b}} d b
$$

For $t_{0} \leq t \leq t_{k}, r_{i}=1(i \geq m, m \leq k)$, the test sample was 1 , failure data of acquisition was 1 , so the likelihood function was:

$$
L\left(1, p_{i}\right)=p_{i}
$$

According to the type (7), the Bayesian estimation of failure probability $p_{i}$ of the prior distribution of was following as:

$$
p_{i}\left(r_{i}=1\right)=\frac{\int_{1}^{C} \int_{p_{i-1}}^{1} \frac{p_{i}^{2}\left(1-p_{i}\right)^{b^{-1}}}{B(1, b)\left(1-p_{i-1}\right)^{b}} d p_{i} d b}{\int_{1}^{C} \int_{p_{i-1}}^{1} \frac{p_{i}\left(1-p_{i}\right)^{-1}}{B(1, b)\left(1-p_{i-1}\right) b} d p_{i} d b}=1-\left(1-p_{i-1}\right) \frac{(C-1) p_{i-1}+2\left(1-p_{i-1}\right) \ln \frac{C+2}{3}-\ln \frac{1+C}{2}}{(C-1) p_{i-1}+\left(1-p_{i-1}\right) \ln \frac{1+C}{2}}
$$

If failure probability $p_{i}$ was calculated for each time, the reliability parameters of special CNC machine have been estimated by weighted least squares, and then the failure probability function could be determined.

\section{Conclusion}

(1) Based on the failure data, in this paper, the determination method of failure rate function of the single sample special NC machine tool has been discussed.

(2) Set up failure data analysis model of a single sample special NC machine tool.

(3) In the case of single failure data and smooth data, how to determine the single sample failure rate function of special NC machine tools, the reliability analysis of the machine tool has yet to be further studied.

\section{References}

[1] Yazhou J, Holim W, Zhixin J.Probability distribution of machining center failures, Reliability Engineering and System Safety, 50(1995) 121-125.

[2] Guo Yong ji, Principles of Reliability Engineering, Tsinghua University Press, Beijing, 2002.

[3] Wang Y Q, Jia Y Z, Yu J Y, et al, Failure database of CNC Laths, Internatinoal Journal of Quality and Reliability Management, 16(1999) 330-340

[4] Ming Han, Reliability Analysis of No Failure Data, China Statistics Press, Beijing, 1999. 